Virginia Commonwealth University

VCU Scholars Compass

1997

\title{
Atomic and electronic structures of neutral and charged boron and boron-rich clusters
}

J. Niu

Virginia Commonwealth University

B. K. Rao

Virginia Commonwealth University

P. Jena

Virginia Commonwealth University, pjena@vcu.edu

Follow this and additional works at: http://scholarscompass.vcu.edu/phys_pubs

Part of the Physics Commons

Niu, J., Rao, B. K., \& Jena, P. Atomic and electronic structures of neutral and charged boron and boron-rich clusters. The Journal of Chemical Physics, 107, 132 (1997). Copyright (C) 1997 American Institute of Physics.

\section{Downloaded from}

http://scholarscompass.vcu.edu/phys_pubs/141

This Article is brought to you for free and open access by the Dept. of Physics at VCU Scholars Compass. It has been accepted for inclusion in Physics Publications by an authorized administrator of VCU Scholars Compass. For more information, please contact libcompass@vcu.edu. 


\title{
Atomic and electronic structures of neutral and charged boron and boron-rich clusters
}

\author{
J. Niu, B. K. Rao, and P. Jena \\ Physics Department, Virginia Commonwealth University, Richmond, Virginia 23284-2000
}

(Received 29 July 1996; accepted 28 March 1997)

\begin{abstract}
Ab initio molecular orbital theory based on both density functional formalism and quantum chemical methods has been used to calculate the equilibrium geometries, binding energies, ionization potentials, fragmentation patterns, and electronic structures of neutral and charged boron clusters containing up to six atoms. Calculations have also been performed on restricted geometries for $\mathrm{B}_{n} \mathrm{X}(n=1,5,12 ; \mathrm{X}=\mathrm{Be}, \mathrm{B}, \mathrm{C})$ and $\mathrm{B}_{20}$ clusters to see if clusters can be designed so as to increase their stability. Energetics of doubly charged $\mathrm{B}_{n}^{++}$clusters have also been studied to find the critical size for Coulomb explosion. The results are compared with existing experimental and theoretical data. (C) 1997 American Institute of Physics. [S0021-9606(97)01525-0]
\end{abstract}

\section{INTRODUCTION}

The realization that atomic clusters constitute a new phase of matter has created considerable interest in the study of their structural and electronic properties. ${ }^{1}$ In the past several years considerable progress has been made in our understanding of the evolution of equilibrium geometries, nature of bonding, cohesive energies and electronic structure of semi-conductors (mostly carbon and silicon), metal, and van der Waals clusters. However, the number of experimental and theoretical studies of boron clusters has been rather limited. This is surprising because boron exhibits some of the most interesting chemistry of all elements in the periodic table. ${ }^{2}$ In addition, boron and boron-rich materials have important technological applications such as in explosives, refractory materials, high modulus fiber composites, stable chemical insulators, high temperature semiconductor devices, and thermoelectric power conversion.

Boron is isovalent with $\mathrm{Al}$, yet the atomic and electronic structure of the two elements is entirely different. $\mathrm{Al}$ is a nearly free-electron metal where the valence electrons are delocalized and the solid phase is face centered cubic. Boron-rich solids, on the other hand, are composed of 12atom clusters of boron having the structure of an icosahedron. $^{2}$ The electronic structure is characterized by three-center bonding, where three boron atoms share a common pool of charge. It is well known that in covalently bonded materials, the bonding charges are centered between two atoms. Thus the electronic structure of boron is intermediate between covalent and metallic bonding. This behavior renders boron-rich solids some of the most unusual properties. For example, boron-rich solids range from conducting materials $\left(\mathrm{B}_{1-x} \mathrm{C}_{x}\right)$ to insulating materials $\left(\mathrm{B}_{12} \mathrm{P}_{2}\right)$.

It is interesting to ask if the unique electronic structures of boron and boron-rich solids are prevalent even in the cluster phase. For example, consider the $\mathrm{B}_{20}$ cluster. Since boron is trivalent, and a dodecahedron is composed of pentagonal faces with each atom being three-fold coordinated, a covalently bonded $\mathrm{B}_{20}$ cluster could exhibit unusual stability like that of $\mathrm{C}_{60}$. It has also been suggested ${ }^{3}$ that $\mathrm{B}_{36} \mathrm{~N}_{24}$ could have a fullerene structure and exhibit marked stability in analogy with $\mathrm{C}_{60}$. However, in experiments where boron clusters were produced by laser ablation of hexagonal boron nitride, the existence of $\mathrm{BN}$ and $\mathrm{B}_{2} \mathrm{~N}$ as the only heteroatomic species was observed. ${ }^{3}$ In contrast, an earlier experiment ${ }^{4}$ had detected the existence of $\mathrm{B}_{n} \mathrm{~N}_{m}^{+}$for various combinations of $n$ and $m$ for $n=2-17$.

Only a handful of experimental investigations on pure boron clusters have been carried out in the past few years. Berkowitz and Chupka ${ }^{5}$ were the first to study the mass spectra of $\mathrm{B}_{2-5}$ clusters. Recently, Hanley et al. ${ }^{6}$ have measured the appearance potentials and fragmentation patterns by studying the collision-induced dissociation of boron cluster ions containing up to 13 atoms. They found that the stabilities generally increase with increasing cluster size, although there are large fluctuations. They also found that clusters smaller than six atoms fragment preferentially by losing a $\mathrm{B}^{+}$, while for larger clusters, the charge remains on the $\mathrm{B}_{n-1}^{+}$fragment.

On the theoretical side, there have been very few studies. Langhoff and Bauschlicher ${ }^{7}$ carried out a calculation of spectroscopic constants of $\mathrm{B}_{2}$ by using an extensive Gaussian basis set and multireference configuration interaction (MRCI) approach. Hanley et al. ${ }^{6}$ and Ray et al. $^{8}$ have calculated the relative stability of small $\mathrm{B}_{n}$ and $\mathrm{B}_{n}^{+}$clusters, as well as their fragmentation pattern and ionization potential. These calculations have been carried out by confining the structures to certain fixed geometries and optimizing the corresponding geometrical parameters. The atomic functions were represented by a less extensive set of Gaussians. $A b$ initio molecular dynamics studies ${ }^{9}$ have also been reported for neutral boron clusters. However, no studies, theoretical or experimental, are available, to our knowledge, on the doubly charged boron clusters. It is interesting to ask if small doubly charged clusters of boron, due to their special chemistry, may be stable against Coulomb explosion.

In this paper we present a comprehensive study of the equilibrium geometries, vertical and adiabatic ionization potentials, and the fragmentation patterns of $\mathrm{B}_{2-6}$ clusters in neutral and singly charged states. The energetics and equilibrium geometries of $\mathrm{B}_{n}^{++}(n=2-6)$ provide insight into 
the role of boron's special chemistry on Coulomb explosion. We have also studied the stability of boron-rich clusters $\left(\mathrm{B}_{n} \mathrm{X} ; n=1,5,12 ; \mathrm{X}=\mathrm{Be}, \mathrm{C}\right)$ to examine if they differ from the corresponding behavior in $\mathrm{Al}$ based clusters. Finally, the energetics of $\mathrm{B}_{20}$ confined to a dodecahedral shape was studied to see if two-center bonding between boron atoms is preferred in the cluster, thus enhancing its relative stability. These calculations are based on $a b$ initio self consistent field molecular orbital theory using both density functional and quantum chemical approaches. In Sec. II we provide a brief discussion of our methods. The results obtained using different levels of theory are presented in Sec. III and summarized in Sec. IV.

\section{THEORETICAL METHODS}

For calculation of the total energies, electronic structure, and equilibrium geometries, we have used the Hartree-Fock method, followed by a correlation correction though MöllerPlesset fourth order perturbation theory (MP4). ${ }^{10}$ This approach is computationally demanding and has been used to study small clusters only. On the other hand, the density functional method with generalized gradient approximation $(\text { GGA })^{11}$ enables one to treat larger clusters not possible within the traditional quantum chemistry formalism. This formulation has been shown ${ }^{11}$ to yield accurate results when compared to experiments in bulk metallic systems. However, a quantitative comparison between GGA and quantum chemical approach in bulk systems is not feasible. Such comparisons can be readily made in small atomic clusters. Here we have used both the methods to study clusters of up to six boron atoms. In the density functional approach we have used the GGA under the B3LYP scheme. ${ }^{12}$ For all calculations, the GAUSSIAN 94 software $^{12}$ has been used. We have made use of the same Gaussian basis functions in both the approaches. For studies of boron-rich clusters $\left(\mathrm{B}_{n} \mathrm{X}\right)(n$ $=1,5,12 ; \mathrm{X}=\mathrm{Be}, \mathrm{B}, \mathrm{C})$, only the density functional approach has been used.

The results also depend on the choice of basis sets. While very extensive basis function containing up to $f$ orbitals, as used by Langhoff and Bauschlicher, ${ }^{7}$ can yield quantitatively accurate results, it cannot be used for a large variety of systems, as studied here. On the other hand, reliable theoretical results can be obtained with less extensive basis sets. We have used $(10 s, 5 p, 1 d / 3 s, 2 p, 1 d)$ basis functions for boron (see Table I) and the 6-311G** basis $(11 s, 5 p, 1 d / 4 s, 3 p, 1 d)$ (Ref. 12) for both beryllium and carbon atoms.

In order to assess the accuracy of the basis functions, we have computed the total energy and ionization potential of $\mathrm{C}$ and $\mathrm{B}$ atoms as well as the preferred spin multiplicity, binding energies, bond lengths, and adiabatic ionization potentials of the respective dimers. These results, obtained at the HF-MP4 (SDTQ) and B3LYP levels of theory, are presented in Table II and compared with available experiment. ${ }^{13}$ Note that the agreement between the experiment and both levels of theory is very good indeed. This provides confidence not only on the levels of theory, but also on the choice of the
TABLE I. Basis set for boron.

\begin{tabular}{|c|c|c|c|c|c|}
\hline \multicolumn{2}{|c|}{ S 71.0} & \multicolumn{2}{|c|}{ P 41.0} & \multicolumn{2}{|c|}{ D 10.9} \\
\hline 2788.41 & 0.001288 & 11.3413 & 0.017988 & 1.488 & 1.0 \\
\hline 419.039 & 0.009835 & 2.43599 & 0.110343 & & \\
\hline 96.4683 & 0.047648 & 0.68358 & 0.383072 & & \\
\hline 28.0694 & 0.160069 & 0.21336 & 0.647895 & & \\
\hline 9.37597 & 0.364984 & \multirow{3}{*}{\multicolumn{2}{|c|}{ P 11.17}} & & \\
\hline 3.40623 & 0.433582 & & & & \\
\hline 1.30566 & 0.140082 & & & & \\
\hline & & 0.200114 & 1.0 & & \\
\hline \multicolumn{2}{|c|}{ S 21.0} & & & & \\
\hline 3.40623 & -.17933 & & & & \\
\hline 0.32448 & 1.062594 & & & & \\
\hline \multicolumn{2}{|c|}{ S 10.9} & & & & \\
\hline 0.10219 & 1.0 & & & & \\
\hline
\end{tabular}

basis sets. In the following section we discuss the results on neutral and charged boron and boron-rich clusters separately.

\section{RESULTS}

Using the theoretical procedure outlined in the previous section, our first task has been to determine the equilibrium geometries of clusters and their corresponding binding energies and electronic structures. We have used the method of steepest descent for this purpose. The cluster was initially constructed by placing the atoms at random locations and calculating the total energy. The forces at the atomic sites were computed by using the numerical gradient technique and the atoms were moved to a new location along the path dictated by the steepest descent method. The process was continued until the forces vanished at every atomic site. It should be emphasized that during this process the cluster could be trapped at various metastable minima that exist on the potential energy hyper surface. It was, therefore, necessary to repeat the above process with different initial geometries. Harmonic vibrational frequencies were calculated at the minimum energy configurations to ensure that the minimization procedure had worked properly. It should also be emphasized that one needs to optimize the spin multiplicities of the clusters as well. In what follows, we only quote the results relevant to the most stable geometries and spin multiplicities for $\mathrm{B}_{n}(n \leqslant 6)$ clusters. For clusters containing 13 atoms and larger, the optimization procedure has been limited in scope and will be discussed as appropriate.

\section{A. Neutral boron clusters}

In Fig. 1 we present the equilibrium geometries as well as the geometries of some isomers of $\mathrm{B}_{2-6}$ clusters calculated using the MP4 level of theory. Results for most of these geometries were also tested out using the GGA level of theory. The corresponding bond distances and bond angles are given in Table III. The preferred spin multiplicity and total energies $\left(E_{n}\right)$ are given in Table IV. The binding energy $E_{b}$ of a cluster of $n$ atoms is calculated from the equation,

$$
E_{b}(n)=-\left[E(n)-n E_{0}\right],
$$


TABLE II. Testing the accuracy of the basis functions using different levels of theory.

\begin{tabular}{|c|c|c|c|c|c|c|c|c|c|c|}
\hline \multirow[b]{2}{*}{ System } & \multirow[b]{2}{*}{ Multiplicity } & \multicolumn{3}{|c|}{ Binding energy $(\mathrm{eV})$} & \multicolumn{3}{|c|}{ Bond length $(\AA)$} & \multicolumn{3}{|c|}{ I.P. $(\mathrm{eV})$} \\
\hline & & MP4 & B3LYP & Expt. & MP4 & B3LYP & Expt. & MP4 & B3LYP & Expt. \\
\hline B & 2 & & & & & & & 8.25 & 8.295 & 8.298 \\
\hline C & 3 & & & & & & & 11.04 & 12.12 & 11.26 \\
\hline $\mathrm{B}_{2}$ & 3 & 2.85 & 2.91 & $2.833 \pm 0.238$ & 1.55 & 1.56 & 1.59 & 10.16 & 9.81 & 10.4 \\
\hline
\end{tabular}

where $E_{0}$ is the energy of the free atom. These are also listed in Table IV for both levels of theory.

Before commenting on the individual geometries and their evolution, we want to compare the results obtained using MP4 and B3LYP techniques. Note that the equilibrium geometries (with the exception of $\mathrm{B}_{5}$ ) and spin multiplicities of clusters using both the methods agree very well with each other wherever comparisons were made. The geometrical parameters (bond lengths and bond angles) and the binding energies are also in good agreement with each other. Systematic increase in binding energy as a function of the cluster size is observed in both the methods. This can be clearly seen from Fig. 2. Note that unlike alkali metal clusters, there are no magic numbers (i.e., clusters with unusual stability) in $B_{n}$ clusters in the size range studied.

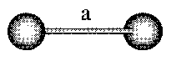

(1)

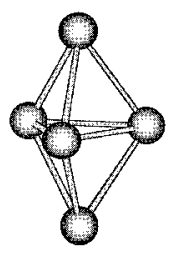

(4a)

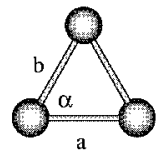

(2)

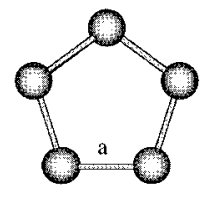

(4b)

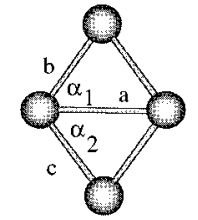

(3)

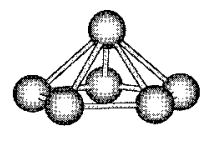

(5a)

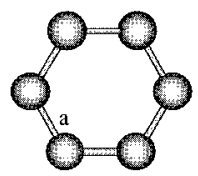

(5b)

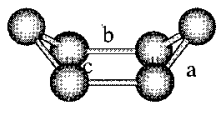

$(5 d)$

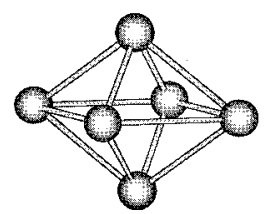

$(5 c)$

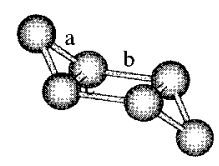

$(5 \mathrm{e})$
FIG. 1. Equilibrium geometries and geometries of isomers of some neutral $\mathrm{B}_{n}(2 \leqslant n \leqslant 6)$ clusters.
We now discuss individual geometries. $\mathrm{B}_{2}$ is the most theoretically studied cluster. Although very early calculations ${ }^{14}$ of $\mathrm{B}_{2}$ predicted the ground state to be in a spin quintet state, later calculations of Dupuis and $\mathrm{Liu}^{15}$ and Langhoff and Bauschlicher ${ }^{7}$ have conclusively shown that the ground state is a spin triplet-in agreement with the experiment. ${ }^{16}$ Our results based upon both MP4 and B3LYP also yield the ground state of $\mathrm{B}_{2}$ to be spin triplet. The bond length and binding energy, $E_{b}(n)$ of $\mathrm{B}_{2}$ computed by Langhoff and Bauschlicher ${ }^{7}$ using up to $f$ and $g$ orbitals in the basis set are, respectively, $1.60 \AA$ and $2.78 \mathrm{eV}$. Our results at the MP4 level of theory for these quantities are $1.55 \AA$ and $2.85 \mathrm{eV}$. This agreement further proves that the basis set we have chosen is adequate to obtain numerically reliable results on larger clusters.

The structure of $\mathrm{B}_{3}$ is an equilateral triangle with a binding energy/atom $\left[E_{b}(n) / n\right]$ of $2.82 \mathrm{eV}$ and bond length 1.52 $\AA$ at the MP4 level of theory. This is in agreement with the results obtained by Ray et al. ${ }^{8}$ as well as by Hanley et al. ${ }^{6}$ Our results, however, differ significantly from those of Ray et al. for $\mathrm{B}_{4}$. These authors found $\mathrm{B}_{4}$ to be a linear chain with a binding energy of $5.02 \mathrm{eV} /$ atom. This is significantly higher than the $3.02 \mathrm{eV} /$ atom binding energy/atom these authors have calculated for $\mathrm{B}_{3}$. The equilibrium structure for $\mathrm{B}_{4}$ in our calculations is a rhombus with a binding energy that is only $0.8 \mathrm{eV}$ per atom higher than the corresponding $\mathrm{B}_{3}$ binding energy. This important discrepancy can only be attributed to a less extensive choice of the basis functions (3-21G*) made by Ray et al., ${ }^{8}$ as we both use the MP4 level of theory. It should be noted that Hanley et al. ${ }^{6}$ did find $\mathrm{B}_{4}$ to be a rhombus. There is only a marginal difference in the bond length and angle between their and our calculations. We also agree with the ground state multiplicity (singlet) of $\mathrm{B}_{4}$ as obtained by these authors. Note that the basis function $\left(6-31 \mathrm{G}^{*}\right)$ used by Hanley et al., ${ }^{6}$ although not as good as the one used here, is superior to that used by Ray et al. ${ }^{8}$

For the $\mathrm{B}_{5}$ we have identified two isomers among which the triangular bipyramid is the most stable structure using the MP4 theory. The bond distance is $1.59 \AA$, which is very close to the values in previous structures. However, the B3LYP predicts the pentagonal structure to be the preferred geometry of $\mathrm{B}_{5}$, although by a small margin. Note that this is the only structure where the B3LYP result is at variance with the MP4 result. The equilibrium structure of $\mathrm{B}_{6}$ is a pentagonal pyramid. This is particularly interesting since the number of bonds in an octahedral structure, Fig. $1(5 \mathrm{c})$, is 12 while it is only 10 in the pentagonal pyramid structure. As Table IV shows, the energy of $\mathrm{B}_{6}$ in the octahedral structure is $2 \mathrm{eV}$ 
TABLE III. Geometrical parameters for neutral $\mathrm{B}_{n}$ clusters (see Fig. 1). For geometries 4(a), 5(a), 5(c), $a$ is the distance from the center of the horizontal plane to the atoms in the plane, and $b$ is the height of the apex atoms from this point. $\alpha$ 's are the angles between two lines in the same plane and $\beta$ 's are the dihedral angles between two planes.

\begin{tabular}{|c|c|c|c|c|c|}
\hline \multirow{2}{*}{$\begin{array}{l}\text { Type of } \\
\text { clusters }\end{array}$} & \multirow[b]{2}{*}{ Geometry } & \multicolumn{2}{|c|}{ Bond lengths $(\AA)$} & \multicolumn{2}{|c|}{ Bond angles $\left(^{\circ}\right)$} \\
\hline & & MP4 & B3LYP & MP4 & B3LYP \\
\hline $\mathrm{B}_{2}$ & Fig. 1-(1) & $a=1.55$ & $a=1.56$ & & \\
\hline $\mathrm{B}_{3}$ & Fig. 1-(2) & $a=1.52, b=1.52$ & $a=1.52, b=1.52$ & $\alpha=60.0$ & $\alpha=60.0$ \\
\hline \multirow[t]{2}{*}{$\mathrm{B}_{4}$} & Fig. 1-(3) & $\begin{array}{l}a=1.79, b=1.51, \\
c=1.51\end{array}$ & $\begin{array}{l}a=1.82, b=1.51, \\
c=1.51\end{array}$ & $\begin{array}{l}\alpha_{1}=53.6, \alpha_{2}=53.6 \\
\beta=180.0\end{array}$ & $\begin{array}{l}\alpha_{1}=52.8, \alpha_{2}=52.8 \\
\beta=180.0\end{array}$ \\
\hline & Fig. 1-(4a) & $a=1.09, b=1.16$ & $a=1.09, b=1.16$ & & \\
\hline \multirow[t]{3}{*}{$\mathrm{B}_{5}$} & Fig. 1-(4b) & $a=1.53$ & $a=1.58$ & & \\
\hline & Fig. 1-(5a) & $a=1.39, b=0.89$ & $a=1.37, b=0.92$ & & \\
\hline & Fig. 1-(5b) & $a=1.52$ & $a=1.52$ & & \\
\hline \multirow[t]{3}{*}{$\mathrm{B}_{6}$} & Fig. 1-(5c) & $a=1.36, b=0.98$ & & & \\
\hline & Fig. 1-(5d) & $\begin{array}{l}a=1.49, b=4.63 \\
c=1.57\end{array}$ & & $\beta=90.1$ & \\
\hline & Fig. 1-(5e) & $\begin{array}{l}a=1.53, b=3.00 \\
c=1.60\end{array}$ & & $\beta=84.1$ & \\
\hline
\end{tabular}

above the ground state structure. The "boat" and the "chair" forms \{see Fig. $1[(5 \mathrm{~d})$ and $(5 \mathrm{e})]\}$ are also of higher energy. This provides a signature of the special electronic structure of boron. If the bonding in $\mathrm{B}_{n}$ clusters would have been a two-centered covalent bond, the octahedral structure would have been the ground state. It is also evident that the seed for an icosahedric growth pattern is shown at the $\mathrm{B}_{6}$ cluster stage. We can conclude that the special chemistry that governs the electronic structure of boron-rich solids is also present in small boron clusters.

\section{B. Singly charged boron clusters}

As an electron is removed from a cluster, the redistribution of charges causes the neutral structure to relax. The difference in the total energy between the neutral and cationic cluster in their respective ground states is the adiabatic ionization potential and has been measured by Hanely et $a l^{6}$ for $\mathrm{B}_{2-13}$ clusters. They have also observed that the fragmen- tation of $\mathrm{B}_{n}^{+}$clusters for $n<6$ proceeds by the emission of a $\mathrm{B}^{+}$atom, while in larger clusters the charge resides on the $\mathrm{B}_{n-1}^{+}$fragment.

We have optimized the geometries of $\mathrm{B}_{n}^{+}(n \leqslant 6)$ clusters both at the MP4 and the B3LYP level of theory. Both methods, once again, yield identical geometries and spin multiplicities as in the case of the neutral clusters. These are presented in Fig. 3. The relevant geometrical parameters are tabulated in Table V. First, we compare the structures of neutral and corresponding cationic clusters. There is very little change among the geometries of neutral and charged dimers, trimers, and tetramers. Significant changes, however, occur in $\mathrm{B}_{5}$ and $\mathrm{B}_{6}$ clusters when an electron is removed. The planar structures are preferred for the positively charged clusters. The primary B-B bond, however, remains very close to that in the neutral clusters in all cases.

In Table VI we compare the total energy and the binding energy/atom $\left[E_{b}^{+}(n) / n\right]$ of $\mathrm{B}_{n}^{+}$clusters obtained using both the theoretical methods. The binding energy of singly

TABLE IV. Energetics and preferred spin multiplicities of neutral $\mathrm{B}_{n}$ clusters using MP4 and B3LYP theories. See Fig. 1 for corresponding geometries. For $\mathrm{B}_{5}$ and $\mathrm{B}_{6}$ clusters, the binding energy/atom is computed only for ground state structures

\begin{tabular}{|c|c|c|c|c|c|c|c|}
\hline \multirow[b]{2}{*}{ Compound } & \multicolumn{2}{|c|}{ Multiplicity } & \multirow[b]{2}{*}{ Geometry } & \multicolumn{2}{|c|}{ Total energy, a.u. } & \multicolumn{2}{|c|}{ Binding energy/atom, eV } \\
\hline & MP4 & B3LYP & & MP4 & B3LYP & MP4 & B3LYP \\
\hline B & 2 & 2 & l & -24.5487 & -24.6398 & I & I \\
\hline $\mathrm{B}_{2}$ & 3 & 3 & Fig. 1-(1) & -49.2022 & -49.3866 & 1.43 & 1.45 \\
\hline $\mathrm{B}_{3}$ & 2 & 2 & Fig. 1-(2) & -73.9567 & -74.2669 & 2.82 & 3.15 \\
\hline \multirow[t]{2}{*}{$\mathrm{B}_{4}$} & 1 & 1 & Fig. 1-(3) & -98.7271 & -99.1168 & 3.62 & 3.79 \\
\hline & & & Fig. 1-(4a) & -123.4108 & -123.8438 & 3.63 & \\
\hline \multirow[t]{3}{*}{$\mathrm{B}_{5}$} & 2 & 2 & Fig. 1-(4b) & -123.3697 & -123.8731 & & 3.67 \\
\hline & & & Fig. 1-(5a) & -148.0944 & -148.7722 & 3.64 & 4.23 \\
\hline & & & Fig. 1-(5b) & -148.0298 & -148.6512 & & \\
\hline \multirow[t]{3}{*}{$\mathrm{B}_{6}$} & 1 & 1 & Fig. 1-(5c) & -148.0199 & & & \\
\hline & & & Fig. 1-(5d) & -148.0445 & & & \\
\hline & & & Fig. 1-(5e) & -148.0199 & & & \\
\hline
\end{tabular}




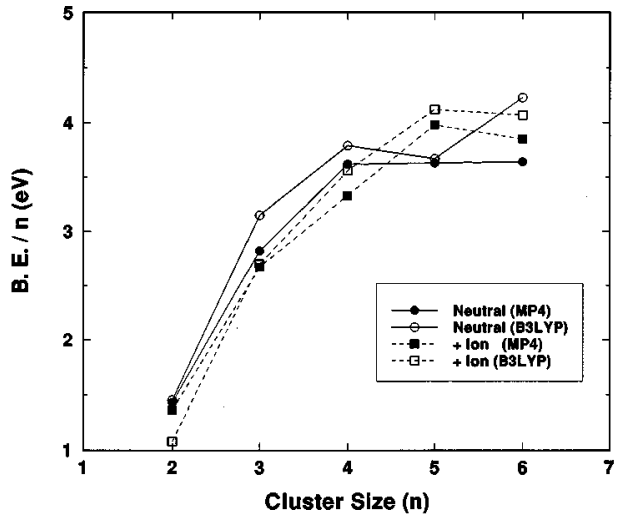

FIG. 2. Comparison of the binding energy/atom, $\left[E_{b}(n) / n\right]$, for neutral and charged $\mathrm{B}_{n}$ clusters calculated using MP4 and B3LYP theories.

charged clusters, $E_{b}^{+}(n)$ is calculated by using the equation

$$
E_{b}^{+}(n)=-\left[E^{+}(n)-(n-1) E_{0}-E_{0}^{+}\right],
$$

where $E^{+}(n)$ and $E_{0}^{+}$are, respectively, the total energies of the $\mathrm{B}_{n}^{+}$and $\mathrm{B}^{+}$in their ground state configurations. Note that both MP4 and B3LYP methods provide the same systematic variation, namely a monotonic rise with cluster size (see Fig. 2). On the contrary, in charged alkali metal clusters, these energies fluctuate with size.

The adiabatic potentials have been calculated by taking the difference between the ground state energies of neutral and positively charged clusters. These values obtained using the MP4 and B3LYP methods are compared with experimental results in Fig. 4. Note that unlike the binding energies in Fig. 2, the adiabatic potentials vary monotonically with size. Although our calculated values at both levels of theory are closer to the experiment than those calculated by Hanley et al. ${ }^{6}$ the agreement is far from perfect.

We next discuss the fragmentation of singly charged $\mathrm{B}_{n}^{+}$clusters. We only deal with binary fragmentation. There are two possible channels: $\mathrm{B}_{n}^{+} \rightarrow \mathrm{B}_{m}^{+}+\mathrm{B}_{n-m}$ or $\mathrm{B}_{n}^{+} \rightarrow \mathrm{B}_{m}$ $+\mathrm{B}_{n-m}^{+}$. The simplest way to examine this is to calculate the dissociation energies,

$$
\Delta E_{n m}^{+}=\left[\begin{array}{c}
{\left[E(m)+E^{+}(n-m)\right]-E^{+}(n)} \\
\text { or } \\
{\left[E^{+}(m)+E(n-m)\right]-E^{+}(n)}
\end{array}\right] .
$$

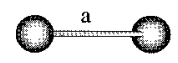

(1)

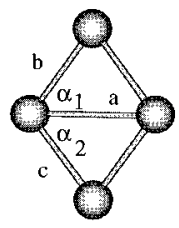

(3)

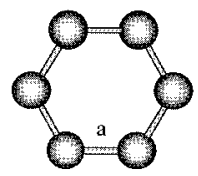

(5a)

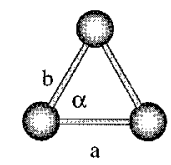

(2)

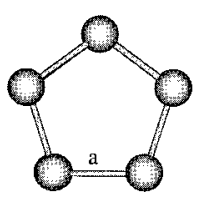

(4)

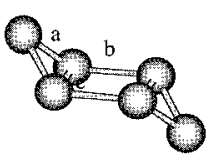

(5b)
FIG. 3. The equilibrium geometries of $\mathrm{B}_{n}^{+}$clusters.

We compare the dissociation energies for different channels with experiment as well as the calculated values of Hanley et al. ${ }^{6}$ in Table VII. The preferred channel is considered as the one for which $\Delta E^{+}$is minimum. In practice, the energy barriers separating the two fragments can also play an important role in the dissociation of the charged clusters. Such calculations have not been performed here. From the energetics in Table VII we see that the preferred channel is the emission of a $\mathrm{B}^{+}$ion for $\mathrm{B}_{2}^{+}$to $\mathrm{B}_{5}^{+}$clusters. For $\mathrm{B}_{6}^{+}$clusters, the preferred channel is the emission of a neutral boron atom. This trend is in agreement with experiment. However, our calculated dissociation energies (see Fig. 5) for the preferred channels, although following experimental systematics, do not agree quantitatively with experiment. ${ }^{6}$

\begin{tabular}{|c|c|c|c|c|c|}
\hline \multirow[b]{2}{*}{ Type of clusters } & \multirow[b]{2}{*}{ Geometry } & \multicolumn{2}{|c|}{ Bond lengths $(\AA)$} & \multicolumn{2}{|c|}{ Bond angles $\left({ }^{\circ}\right)$} \\
\hline & & MP4 & B3LYP & MP4 & B3LYP \\
\hline $\mathrm{B}_{2}^{+}$ & Fig. 3-(1) & $a=1.75$ & $a=1.80$ & & \\
\hline $\mathrm{B}_{3}^{+}$ & Fig. 3-(2) & $a=1.60, b=1.60$ & $a=1.55, b=1.55$ & $\alpha=60.0$ & $\alpha=60.0$ \\
\hline $\mathrm{B}_{4}^{+}$ & Fig. 3-(3) & $a=1.98, b=1.55, c=1.55$ & $\begin{array}{l}a=2.00, b=1.54 \\
c=1.54\end{array}$ & $\begin{array}{l}\alpha_{1}=50.5, \quad \alpha_{2}=50.5 \\
\beta=180.0\end{array}$ & $\begin{array}{l}\alpha_{1}=49.5, \quad \alpha_{2}=49.5 \\
\beta=180.0\end{array}$ \\
\hline $\mathrm{B}_{5}^{+}$ & $\begin{array}{l}\text { Fig. 3-(4) } \\
\text { Fig. 3-(5a) }\end{array}$ & $\begin{array}{l}a=1.57 \\
a=1.55\end{array}$ & $\begin{array}{l}a=1.54 \\
a=1.54\end{array}$ & & \\
\hline $\mathrm{B}_{6}^{+}$ & Fig. 3-(5b) & $a=1.67, b=1.60, c=1.69$ & $\begin{array}{l}a=1.64, b=1.65 \\
c=1.67\end{array}$ & $\beta_{1}=149.2, \beta_{2}=149.2$ & $\beta_{1}=110.1, \beta_{2}=110.1$ \\
\hline
\end{tabular}

TABLE V. Geometrical parameters for $\mathrm{B}_{n}^{+}$clusters (see Fig. 3). The notations for the figures are as given in Table III. 
TABLE VI. Energetics for singly charged boron clusters, $\mathrm{B}_{n}^{+}(n=1-6)$.

\begin{tabular}{|c|c|c|c|c|c|c|c|}
\hline \multirow[b]{2}{*}{ Clusters } & \multicolumn{2}{|c|}{ Multiplicity } & \multirow[b]{2}{*}{ Geometry } & \multicolumn{2}{|c|}{ Total energy, a.u. } & \multicolumn{2}{|c|}{ Binding energy/atom, eV } \\
\hline & MP4 & B3LYP & & MP4 & B3LYP & MP4 & B3LYP \\
\hline $\mathrm{B}_{1}^{+}$ & 1 & 1 & I & -24.2456 & -24.3348 & I & l \\
\hline $\mathrm{B}_{2}^{+}$ & 2 & 2 & Fig. 3-(1) & -48.8941 & -49.0540 & 1.36 & 1.08 \\
\hline $\mathrm{B}_{3}^{+}$ & 1 & 1 & Fig. 3-(2) & -73.6379 & -73.9121 & 2.67 & 2.70 \\
\hline $\mathrm{B}_{4}^{+}$ & 2 & 2 & Fig. 3-(3) & -98.3815 & -98.7779 & 3.33 & 3.56 \\
\hline \multirow[t]{2}{*}{$\mathrm{B}_{5}^{+}$} & 1 & 1 & Fig. 3-(4) & -123.1712 & -123.6511 & 3.98 & 4.12 \\
\hline & & & Fig. 3-(5a) & -147.8376 & -148.4326 & 3.85 & 4.07 \\
\hline $\mathrm{B}_{6}^{+}$ & 2 & 2 & Fig. 3-(5b) & -147.7578 & -148.3328 & & \\
\hline
\end{tabular}

\section{Doubly charged boron clusters}

As a cluster is doubly ionized, the added repulsion between the two positive charges tends to destabilize the cluster. This destabilization force is the strongest for the smallest clusters. The smaller clusters usually spontaneously fragment unless there is an energy barrier that can protect the cluster against such a fate. This phenomenon is known as Coulomb explosion $^{17}$ and has been extensively studied in metal clusters. The critical size for Coulomb explosion increases with the increasing level of ionization. It has been shown theoretically ${ }^{18}$ and verified experimentally ${ }^{19}$ that some of the doubly charged metal dimers could be metastable and the electronic structure could play an important role in determining the metastability.

To our knowledge, no experimental or theoretical work on doubly charged $\mathrm{B}_{n}$ clusters is yet available. Since the chemistry of $\mathrm{B}_{n}$ clusters and solids is rather special, we have investigated the energetics and geometries of $\mathrm{B}_{n}^{++}$clusters $(n \leqslant 6)$ using MP4 theory. In Fig. 6 we give the equilibrium geometries. The corresponding geometrical parameters are given in Table VIII. Note that unlike the singly charged clusters, the doubly charged clusters are all linear chains. However, the length of the bonds in the clusters remain almost unaltered from the neutral dimer value. The innermost bonds are slightly stretched as the excess charge mostly resides at the end atoms.

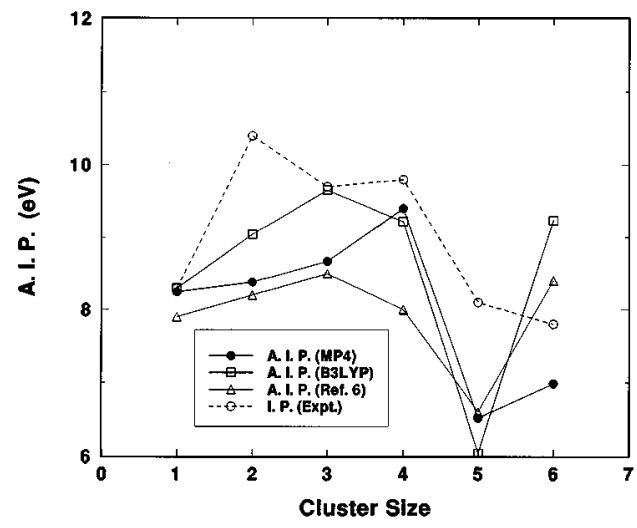

FIG. 4. Comparison of the experimental adiabatic ionization potentials of $\mathrm{B}_{n}$ clusters with those calculated using MP4 and B3LYP theories and other calculations (Ref. 6)
What is more unique about $\mathrm{B}_{n}^{++}$clusters compared to other doubly charged clusters is their stability. To analyze these we have calculated the binding energy of the $\mathrm{B}_{n}^{++}$ cluster using the relation: $E_{b}^{++}(n)=\left[2 E\left(\mathrm{~B}^{+}\right)+(n-2)\right.$ $\left.\times E(\mathrm{~B})-E\left(\mathrm{~B}_{n}^{++}\right)\right]$. In Table IX we give the total energies, binding energies, and spin multiplicities of $\mathrm{B}_{n}^{++}$clusters using the MP4 method. We find that $\mathrm{B}_{2}^{++}$and $\mathrm{B}_{3}^{++}$are unstable against fragmentation, as indicated by negative value of $E_{b}^{++}(n)$, while the larger $\mathrm{B}_{n}^{++}(n \geqslant 4)$ clusters are stable against dissociation into individual ions and neutral atoms.

We have also checked to see if $\mathrm{B}_{n}^{++}$is stable against any one of the two fragmentation channels: $\mathrm{B}_{m}^{+}+\mathrm{B}_{n-m}^{+}$and $\mathrm{B}_{m}^{++}+\mathrm{B}_{n-m}$. This is done by calculating the dissociation energy using the equations:

$$
\begin{aligned}
& \Delta E_{n m}^{++}=E\left(\mathrm{~B}_{n}^{++}\right)-\left[E\left(\mathrm{~B}_{m}^{+}\right)+E\left(\mathrm{~B}_{n-m}^{+}\right)\right], \\
& \Delta \widetilde{E}_{n m}^{++}=E\left(\mathrm{~B}_{n}^{++}\right)-\left[E\left(\mathrm{~B}_{n-m}\right)+E\left(\mathrm{~B}_{m}^{++}\right)\right] .
\end{aligned}
$$

A negative value of $\Delta E_{n m}^{++}$or $\Delta \widetilde{E}_{n m}^{++}$for any value of $n$ and $m$ means that fragmentation in that channel is not preferred. If $\Delta E_{n m}^{++}$and $\Delta \widetilde{E}_{n m}^{++}$are both negative for all possible values of $m$, we can conclude that $\mathrm{B}_{n}^{++}$cluster could be stable against Coulomb explosion. The critical size for Coulomb explosion corresponds to the smallest value of $n$ for which the above statement holds. Using the total energies of $\mathrm{B}_{n}$, $\mathrm{B}_{n}^{+}$, and $\mathrm{B}_{n}^{++}$clusters given in Tables IV, VI, and IX at the MP4 level of theory, we find that none of the doubly charged clusters studied has reached the critical size for Coulomb explosion. This argument does not take into account the fact that energy barriers, as the fragments separate, may have an important influence on the critical size for Coulomb explosion observed experimentally. For example, in some transition metal clusters, doubly charged dimers have been observed, ${ }^{19}$ even though $\Delta E_{n m}^{++}$is negative. ${ }^{18}$ Study of such energy barriers and experiments on the critical size for $\mathrm{B}_{n}^{++}$clusters should yield interesting results.

\section{Compound clusters of boron}

The electronic structure and stability of clusters can be significantly altered by introducing impurities. As mentioned earlier, the structure of $\mathrm{B}_{n-x} \mathrm{C}_{x}$ solids is composed of $\mathrm{B}_{12}$ icosahedra connected by $\mathrm{C}$-atoms, ie., the $\mathrm{C}$-atoms reside outside the icosahedric cage. ${ }^{2}$ It was demonstrated ${ }^{20}$ that $\mathrm{Al}_{12} \mathrm{C}$, with carbon occupying the central site of an icosahe- 
TABLE VII. Dissociation energies of $\mathrm{B}_{n}^{+}$clusters for different channels using MP4 and B3LYP theory.

\begin{tabular}{|c|c|c|c|c|c|}
\hline \multirow[b]{2}{*}{ Cluster } & \multirow[b]{2}{*}{ Dissociation channels } & \multicolumn{2}{|c|}{ D. E. of this work, eV } & \multirow{2}{*}{$\begin{array}{l}\text { D. E. of Hanley's } \\
\text { work (Ref. 6), eV }\end{array}$} & \multirow{2}{*}{$\begin{array}{c}\text { D. E. of experiment, } \\
\mathrm{eV}\end{array}$} \\
\hline & & MP4 & B3LYP & & \\
\hline \multirow[t]{2}{*}{$\mathrm{B}_{2}^{+}$} & $\mathrm{B}_{2}^{+} \Rightarrow \mathrm{B}^{+}+\mathrm{B}$ & 2.715 & 2.150 & 0.7 & $0.8 \pm 0.6$ \\
\hline & $\mathrm{B}_{3}^{+} \Rightarrow \mathrm{B}^{+}+\mathrm{B}_{2}$ & 5.171 & 5.187 & 1.1 & $2.3 \pm 0.6$ \\
\hline \multirow[t]{2}{*}{$\mathrm{B}_{3}^{+}$} & $\mathrm{B}_{3}^{+} \Rightarrow \mathrm{B}_{2}^{+}+\mathrm{B}$ & 5.307 & 5.938 & 2.0 & $4.3 \pm 0.7$ \\
\hline & $\mathrm{B}_{4}^{+} \Rightarrow \mathrm{B}^{2}+\mathrm{B}_{3}$ & 4.874 & 4.793 & 1.2 & $2.4 \pm 0.6$ \\
\hline \multirow[t]{2}{*}{$\mathrm{B}_{4}^{+}$} & $\mathrm{B}_{4}^{+} \Rightarrow \mathrm{B}_{2}^{+}+\mathrm{B}_{2}$ & 7.757 & 9.175 & 3.2 & I \\
\hline & $\mathrm{B}_{4}^{+} \Rightarrow \mathrm{B}_{3}^{+}+\mathrm{B}$ & 5.301 & 6.147 & 2.8 & $8.0 \pm 0.1 .5$ \\
\hline \multirow[t]{6}{*}{$\mathrm{B}_{5}^{+}$} & $\mathrm{B}_{5}^{+} \Rightarrow \mathrm{B}^{+}+\mathrm{B}_{4}$ & 5.399 & 5.426 & 2.0 & $3.6 \pm 0.6$ \\
\hline & $\mathrm{B}_{5}^{+} \Rightarrow \mathrm{B}_{2}^{+}+\mathrm{B}_{3}$ & 8.715 & 8.981 & I & I \\
\hline & $\mathrm{B}_{5}^{+} \Rightarrow \mathrm{B}_{3}^{+}+\mathrm{B}_{2}$ & 9.006 & 9.585 & l & / \\
\hline & $\mathrm{B}_{5}^{+} \Rightarrow \mathrm{B}_{4}^{+}+\mathrm{B}$ & 6.555 & 6.348 & I & $7.1 \pm 0.6$ \\
\hline & $\mathrm{B}_{6}^{+} \Rightarrow \mathrm{B}^{+}+\mathrm{B}_{5}$ & 4.929 & 6.112 & I & $3.2 \pm 0.7$ \\
\hline & $\mathrm{B}_{6}^{+} \Rightarrow \mathrm{B}_{2}^{+}+\mathrm{B}_{4}$ & 5.886 & 7.121 & I & / \\
\hline \multirow[t]{3}{*}{$\mathrm{B}_{6}^{+}$} & $\mathrm{B}_{6}^{+} \Rightarrow \mathrm{B}_{3}^{+}+\mathrm{B}_{3}$ & 6.610 & 6.898 & I & I \\
\hline & $\mathrm{B}_{6}^{+} \Rightarrow \mathrm{B}_{4}^{+}+\mathrm{B}_{2}$ & 6.906 & 7.292 & I & I \\
\hline & $\mathrm{B}_{6}^{+} \Rightarrow \mathrm{B}_{5}^{+}+\mathrm{B}$ & 3.201 & 3.854 & 2.2 & $2.7 \pm 0.6$ \\
\hline
\end{tabular}

dron, is far more stable than $\mathrm{Al}_{13}$. This stability is partly derived from the fact that $\mathrm{Al}_{12} \mathrm{C}$ has 40 valence electrons that are enough to close the outermost electronic shells. Since boron is isoelectronic with aluminum, one wonders if $\mathrm{B}_{12} \mathrm{C}$ with $\mathrm{C}$ occupying the central site can show enhanced stability.

The binding energy of a compound cluster $\mathrm{B}_{n} \mathrm{X}$ is defined as

$$
\widetilde{E}_{b}=E(\mathrm{X})+n E(\mathrm{~B})-E\left(\mathrm{~B}_{n} \mathrm{X}\right) .
$$

The geometries of $\mathrm{B}_{n} \mathrm{X}$ clusters are given in Figures 7(a) and 7 (b), respectively. The corresponding bond lengths, total energies, binding energies, spin multiplicity, and Mulliken charges are given in Table $\mathrm{X}$. We note that out of the three dimers $\mathrm{BBe}, \mathrm{B}_{2}$, and $\mathrm{BC}, \mathrm{BBe}$ is the least bound, while the binding energy of $\mathrm{BC}$ is larger than that of $\mathrm{B}_{2}$. $\mathrm{Be}$ is a closed shell atom and its weak interaction with the $\mathrm{B}$ atom is understandable. However, this changes as we go to larger clusters. For example, the binding energy of $\mathrm{B}_{5} \mathrm{Be}$ is only marginally smaller than that of $\mathrm{B}_{5} \mathrm{~B}$ or $\mathrm{B}_{5} \mathrm{C}$. In $\mathrm{B}_{12} \mathrm{X}$ clusters,

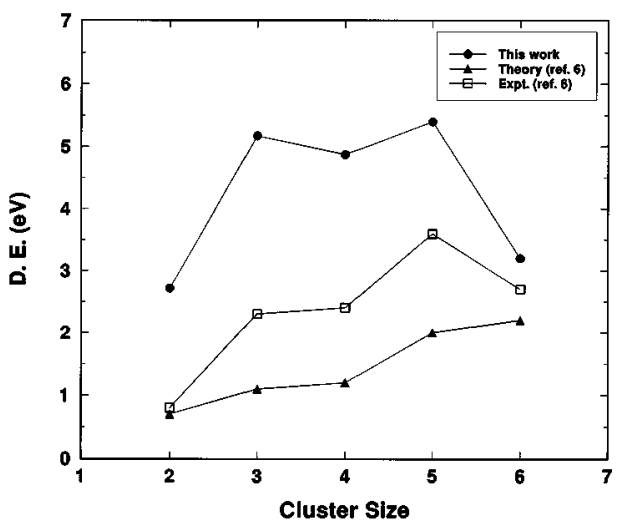

FIG. 5. Comparison of the preferred dissociation channels of $\mathrm{B}_{n}^{+}$clusters calculated using MP4 level of theory with the experimental and other theoretical works (Ref. 6). note that $\mathrm{B}_{12} \mathrm{Be}$ is more strongly bound than either $\mathrm{B}_{12} \mathrm{~B}$ or $\mathrm{B}_{12} \mathrm{C}$. This is particularly striking since the number of valence electrons in $\mathrm{B}_{12} \mathrm{Be}$ is 38 while that in $\mathrm{B}_{12} \mathrm{C}$ is 40 . We recall that $\mathrm{Al}_{12} \mathrm{C}$ has a binding energy that is $4.4 \mathrm{eV}$ larger $^{20}$ than that of $\mathrm{Al}_{13}$. This is due to the fact that 40 electrons correspond to closed electronic shells in an otherwise jellium cluster. Thus $\mathrm{B}_{12} \mathrm{C}$ does not behave like $\mathrm{Al}_{12} \mathrm{C}$. This is fur-

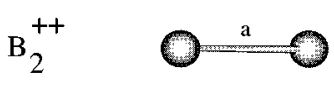

(1)

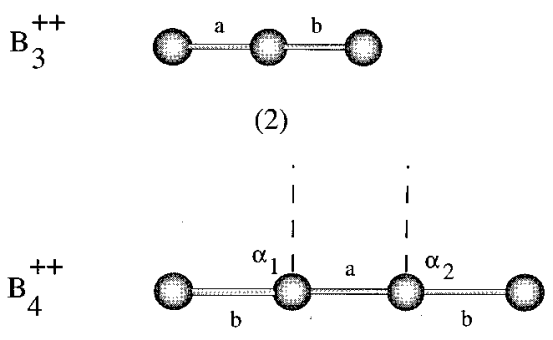

(3)

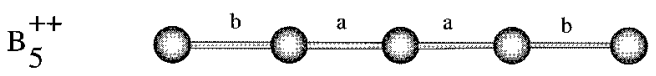

(4)

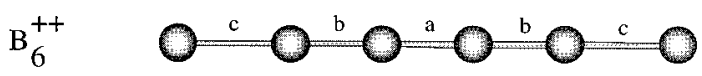

(5)

FIG. 6. Preferred geometries of $\mathrm{B}_{n}^{++}$clusters. 
TABLE VIII. Geometrical parameters for $\mathrm{B}_{n}^{++}$clusters (see Fig. 6).

\begin{tabular}{llcc}
\hline \hline $\begin{array}{c}\text { Type of } \\
\text { clusters }\end{array}$ & Geometry & $\begin{array}{c}\text { Bond lengths } \\
(\AA)\end{array}$ & $\begin{array}{c}\text { Bond angles } \\
\left({ }^{\circ}\right)\end{array}$ \\
\hline $\mathrm{B}_{2}^{++}$ & Fig. 6-(1) & $a=1.54$ & \\
$\mathrm{~B}_{3}^{++}$ & Fig. 6-(2) & $a=1.60, b=1.60$ & \\
$\mathrm{~B}_{4}^{++}$ & Fig. 6-(3) & $a=1.59, b=1.50$ & $\alpha_{1}=89.6, \alpha_{2}=89.6$ \\
$\mathrm{~B}_{5}^{++}$ & Fig. 6-(4) & $a=1.58, b=1.50$ & \\
$\mathrm{~B}_{6}^{++}$ & Fig. 6-(5) & $a=1.53, b=1.67, c=2.15$ & \\
\hline \hline
\end{tabular}

ther indication that the electronic structure of boron clusters is different from its isoelectronic aluminum clusters, just as is the case with the bulk.

Further insight into the electronic structure of boron-rich clusters can be obtained by examining the Mulliken charge distribution in Table $\mathrm{X}$. Note that the net charge on the central $\mathrm{B}$ in $\mathrm{B}_{13}$ icosahedron is negative, as it is on the central $\mathrm{C}$ and $\mathrm{Be}$ site in $\mathrm{B}_{12} \mathrm{X}$ cluster. The magnitude of the charge transfer increases as the central atom changes from $\mathrm{C}$ to $\mathrm{Be}$. This is consistent with the increase in the binding energy.

Finally, we discuss the stability of $\mathrm{B}_{20}$. This structure was confined to the dodecahedral shape (see Fig. 8) and the bond distance was optimized. The objective was to see if two-center bonding can be important in boron clusters. The binding energy/atom of $\mathrm{B}_{20}$ is found to be $5.56 \mathrm{eV}$, which is only marginally larger than that in $\mathrm{B}_{12}$. Since the general tendency of binding energy is to increase with size, this marginal increase is not indicative of any magical stability of $B_{20}$. In other words, $B_{20}$ may not constitute the smallest structure that could resemble a fullerene. We wish to emphasize that the studies of the relative stabilities of compound boron clusters carried out in this section have made use of restricted geometries. For example, the equilibrium structure of $\mathrm{B}_{13}$ cannot be an icosahedron (with the central site occupied), as it is energetically unstable compared to an icosahedric $\mathrm{B}_{12}$ cluster (icosahedron with the central site vacant) plus an isolated boron atom.

\section{CONCLUSIONS}

The energetics and atomic and electronic structure of neutral and charged boron and boron-rich clusters have been studied using self consistent molecular orbital theory and two levels of approximation for the exchange and correlation potential. The results based on generalized gradient approximation agree well with those based on the Hartree-Fock-

TABLE IX. Energetics and preferred spin multiplicities of $\mathrm{B}_{n}^{++}(n=1$ -6) clusters.

\begin{tabular}{ccccc}
\hline \hline Compound & Multiplicity & Geometry & $\begin{array}{c}\text { Total energy, } \\
\text { a.u. }\end{array}$ & $\begin{array}{c}\text { Binding energy, } \\
\text { eV }\end{array}$ \\
\hline $\mathrm{B}_{1}^{++}$ & 2 & $/$ & -23.1461 & $/$ \\
$\mathrm{B}_{2}^{++}$ & 1 & Fig. 6-(1) & -48.1069 & -10.45 \\
$\mathrm{~B}_{3}^{++}$ & 2 & Fig. 6-(2) & -72.9823 & -1.57 \\
$\mathrm{~B}_{4}^{++}$ & 3 & Fig. 6-(3) & -97.6646 & 2.07 \\
$\mathrm{~B}_{5}^{++}$ & 4 & Fig. 6-(4) & -122.4083 & 7.37 \\
$\mathrm{~B}_{6}^{++}$ & 3 & Fig. 6-(5) & -147.2227 & 14.60 \\
\hline \hline
\end{tabular}
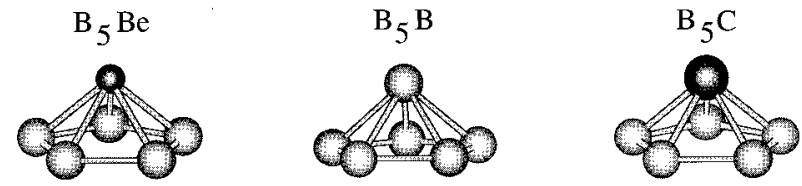

(a)

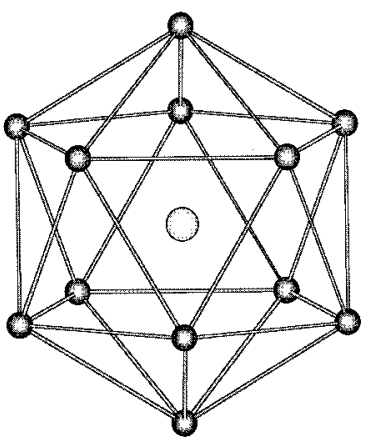

(b)

FIG. 7. (a) Equilibrium geometries of $\mathrm{B}_{5} \mathrm{X}(\mathrm{X}=\mathrm{Be}, \mathrm{B}, \mathrm{C})$ clusters. (b) Icosahedric geometry of $\mathrm{B}_{12} \mathrm{X}$ cluster. The $\mathrm{X}$ atom $(\mathrm{Be}, \mathrm{C})$ is at the center of the icosahedron.

Möller-Plesset method. A number of conclusions are made. (1) The energetics do not reveal the existence of any magic number, as is well known for alkali metal clusters. (2) The nearest neighbor distance in clusters does not differ significantly from the dimer bond length. This is in contrast with the results in metallic or covalent systems. (3) The nature of

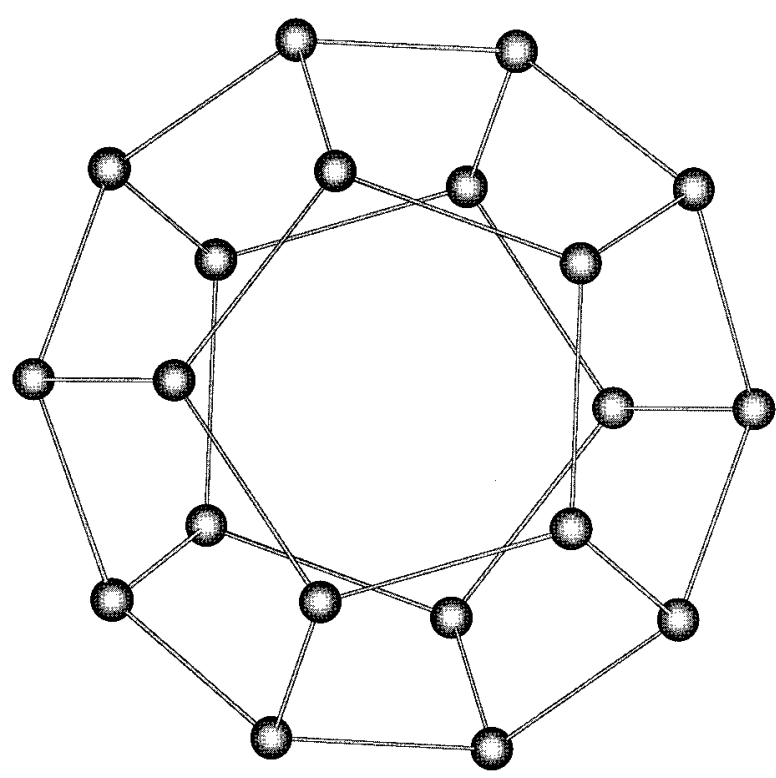

FIG. 8. The dodecahedral geometry of $\mathrm{B}_{20}$. 
TABLE X. Energetics, bond lengths (distance of $X$ from B), preferred spin multiplicities, binding energies, and Mulliken charges in boron-rich clusters and $\mathrm{B}_{20}$ clusters using B3LYP theory. Note that the Mulliken charge on $\mathrm{B}$ represents an average over all $\mathrm{B}$ sites.

\begin{tabular}{ccccccr}
\hline \hline & & & & & \multicolumn{2}{c}{$\begin{array}{c}\text { Mulliken charge distribution } \\
\text { for } \mathrm{B}_{n} \mathrm{X}\end{array}$} \\
\cline { 5 - 7 } $\begin{array}{c}\text { Type of } \\
\text { clusters, } \mathrm{B}_{n} \mathrm{X}\end{array}$ & $\begin{array}{c}\text { Total } \\
\text { energy, eV }\end{array}$ & $\begin{array}{c}\text { Bond } \\
\text { length, } \AA\end{array}$ & Multiplicity & B.E./n, eV & $\mathrm{B}$ & $\mathrm{X}$ \\
\hline $\mathrm{B}_{1} \mathrm{~B}$ & -49.000497 & 1.56 & 3 & 2.178 & 0.00 & 0.00 \\
$\mathrm{~B}_{1} \mathrm{C}$ & -62.224208 & 1.48 & 4 & 3.086 & 0.38 & -0.38 \\
$\mathrm{~B}_{1} \mathrm{Be}$ & -39.016911 & 1.88 & 2 & 1.071 & 0.08 & -0.08 \\
$\mathrm{~B}_{5} \mathrm{~B}$ & -147.714168 & 1.64 & 1 & 5.409 & 0.04 & -0.22 \\
$\mathrm{~B}_{5} \mathrm{C}$ & -160.876570 & 1.66 & 2 & 5.434 & 0.05 & -0.25 \\
$\mathrm{~B}_{5} \mathrm{Be}$ & -137.673035 & 1.70 & 2 & 4.779 & 0.06 & -0.31 \\
$\mathrm{~B}_{12}$ & -295.337812 & 1.78 & 1 & 5.204 & 0.00 & 0.00 \\
$\mathrm{~B}_{12} \mathrm{~B}$ & -319.818887 & 1.99 & 2 & 4.931 & 0.04 & -0.44 \\
$\mathrm{~B}_{12} \mathrm{C}$ & -333.083451 & 2.05 & 3 & 5.156 & 0.09 & -1.06 \\
$\mathrm{~B}_{12} \mathrm{Be}$ & -310.075338 & 1.83 & 1 & 5.263 & 0.11 & -1.34 \\
$\mathrm{~B}_{20}$ & -492.486284 & 2.47 & 1 & 5.553 & 0.00 & 0.00 \\
\hline \hline
\end{tabular}

electronic bonding in boron clusters is similar to that in boron-rich solids and is characterized by a three-center bond. (4) The geometries of singly charged $\mathrm{B}_{5}$ and $\mathrm{B}_{6}$ clusters are significantly different from the corresponding neutral geometries, while in smaller clusters the effect of ionization on geometries is rather minimal. (5) For clusters consisting of fewer than 6 atoms, the fragmentation of $\mathrm{B}_{n}^{+}$proceeds by the emission of a $\mathrm{B}^{+}$ion as seen experimentally. (6) The critical size for Coulomb explosion of doubly charged $\mathrm{B}_{n}^{++}$clusters has not been reached up to $n=6$. (7) The energetics of the compound $\mathrm{B}_{n}$ clusters containing impurities such as $\mathrm{Be}$ and $\mathrm{C}$ suggest that their relative stabilities depend on cluster size and deviate markedly from what can be expected from a simple jellium model. (8) Contrary to initial expectation, $\mathrm{B}_{20}$ does not show any marked stability. This indicates that boron clusters are not stabilized by two-center bonding.

\section{ACKNOWLEDGMENT}

This work was supported in part by a research grant from the U.S. Department of Energy (DEFG05-87ER45316).

${ }^{1}$ Physics and Chemistry of Finite Systems: From Clusters to Crystals, Vols. I and II, edited by P. Jena, S. N. Khanna, and B. K. Rao (Kluwer, Dordrecht, 1992).

${ }^{2}$ D. Emin, Phys. Today 55 (1987); Boron Hydride Chemistry, edited by E.

L. Mutterties (Academic, New York, 1975).

${ }^{3}$ S. J. La Placa, P. A. Roland, and J. J. Wynne, Chem. Phys. Lett. 190, 163 (1992).

${ }^{4}$ S. Becker and H. J. Dietze, Int. J. Mass Spectrom. Ion Processes 73, 157 (1986).
${ }^{5}$ J. Berkowitz and W. A. Chupka, J. Chem. Phys. 40, 2735 (1964).

${ }^{6}$ L. Hanley, J. L. Whitten, and S. L. Anderson, J. Phys. Chem. 92, 5803 (1988).

${ }^{7}$ S. R. Langhoff and C. W. Bauschlicher, J. Chem. Phys. 95, 5882 (1991).

${ }^{8}$ A. K. Ray, I. A. Howard, and K. M. Kanal, Phys. Rev. B 45, 14247 (1992).

${ }^{9}$ R. Kawai, M. W. Sung, and J. H. Weare in Physics and Chemistry of Finite Systems: From Clusters to Crystals, Vol. I, edited by P. Jena, S. N. Khanna, and B. K. Rao (Kluwer, Dordrecht, 1992), p. 441.

${ }^{10}$ W. J. Hehre, L. Radom, P. v. R. Schleyer, and J. A. Pople, Ab Initio Molecular Orbital Theory (Wiley, New York, 1986).

${ }^{11}$ A. D. Becke, Phys. Rev. A 38, 3098 (1988); A. D. Becke, J. Chem. Phys. 84, 4524 (1988); J. P. Perdew and Y. Wang, Phys. Rev. B 45, 13244 (1992) and references therein; A. D. Becke, J. Chem. Phys. 98, 5648 (1993).

${ }^{12}$ Gaussian 94, Revision B 1, M. J. Frisch, G. W. Trucks, H. B. Schlegel, P. M. W. Gill, B. G. Johnson, M. A. Robb, J. R. Cheeseman, T. Keith, G. A. Petersson, J. A. Montgomery, K. Raghavachari, M. A. Al-Laham, V. G. Zakrzewski, J. V. Ortiz, J. B. Foresman, J. Cioslowski, B. B. Stefanov, A. Nanayakkara, M. Challacombe, C. Y. Peng, P. Y. Ayala, W. Chen, M. W. Wong, J. L. Andres, E. S. Replogle, R. Gomperts, R. L. Martin, D. J. Fox, J. S. Binkley, D. J. Defrees, J. Baker, J. P. Stewart, M. Head-Gordon, C. Gonzalez, and J. A. Pople, Gaussian, Inc., Pittsburgh PA, 1995.

${ }^{13}$ A. E. Douglas and G. Herzberg, Can. J. Res. Sect. A 18, 165 (1940).

${ }^{14}$ C. F. Bender and E. R. Davidson, J. Chem. Phys. 46, 3313 (1967).

${ }^{15}$ M. Dupuis and B. Liu, J. Chem. Phys. 68, 2902 (1978).

${ }^{16}$ P. J. Bruna and J. S. Wright, J. Phys. Chem. 94, 1774 (1990).

${ }^{17}$ K. Sattler, J. Mühlbach, O. Echt, P. Pfau, and E. Recknagel, Phys. Rev. Lett. 47, 160 (1981); B. K. Rao, P. Jena, M. Manninen, and R. H. Nieminen, Phys. Rev. Lett. 58, 1188 (1987).

${ }^{18}$ F. Liu, M. R. Press, S. N. Khanna, and P. Jena, Phys. Rev. Lett. 59, 2562 (1987).

${ }^{19}$ T. T. Tsong, Surf. Sci. 177, 593 (1986).

${ }^{20}$ S. N. Khanna and P. Jena, Phys. Rev. B 51, 13705 (1995). 